\title{
THE EPIDEMIOLOGICAL SURVEY TO IDENTIFY THE SIGNS OF TEMPOROMANDIBULAR JOINT DISORDERS IN ASYMPTOMATIC YOUNG INDIVIDUALS ATTENDING A TERTIARY CARE HOSPITAL IN COASTAL KARNATAKA
}

\author{
Ganaraj Shetty ${ }^{1}$ Chethan Hegde 2
}

${ }^{1}$ Senior Lecturer, Department of Prosthodontics, A. B. Shetty Memorial Institute of Dental Sciences, Deralakatte, Mangalore, Karnataka, India.

${ }^{2} H O D$, Department of Prosthodontics, A. B. Shetty Memorial Institute of Dental Sciences, Deralakatte, Mangalore, Karnataka, India. ABSTRACT

\section{BACKGROUND}

Temporomandibular joint disorder refers to a set of a complex disorders that manifest as orofacial pain associated with muscles and temporomandibular joint, thus restricting the jaw movements and other functional activities of facial and masticatory muscles. The literature survey says that $25 \%$ of the population suffers from temporomandibular joint disorders; only $2 \%$ of the population will report to the health professional and seek treatment. The epidemiological survey to identify the signs of temporomandibular joint disorders in asymptomatic young individuals attending a tertiary care hospital in coastal Karnataka, so as to define the presence of the disorder in the said population.

\section{MATERIALS AND METHODS}

Sample sizes of 200 healthy adolescents from age group 15 to 25 years were selected from the Oral Medicine and Radiology Department, A. B. Shetty Memorial Institute of Dental Sciences, Deralakatte, Mangalore. Individuals fulfilling the eligibility criteria were included in the study. Written informed consent was obtained from each individual. The chosen samples were clinically examined using auscultation and palpation method based on Research Diagnostic Criteria Index along with a self-report questionnaire.

\section{RESULTS}

Among 200 subjects, 96 were male, 104 were female. Clicking was observed in $16 \%$ of the study subjects with $79 \%$ of subjects having maximum intercuspation. Deviation, muscle tenderness was observed in $4 \%$ of subjects, pain while yawning and wide opening of mouth was observed in $3 \%$ of subjects.

\section{CONCLUSION}

Based on the results above presented, it was concluded that signs of temporomandibular disorders were present in adolescents. The presence of clinical signs associated with subjective symptoms was confirmed in asymptomatic individuals attending a tertiary care hospital of coastal Karnataka. The temporomandibular joint sounds showed greater prevalence; this finding must be interpreted carefully in order to detect factors that can interfere with proper functioning of stomatognathic system growth and development.

\section{KEY WORDS}

Temporomandibular Joint, Asymptomatic, Temporomandibular Disorder.

HOW TO CITE THIS ARTICLE: Shetty G, Hegde C. The epidemiological survey to identify the signs of temporomandibular joint disorders in asymptomatic young individuals attending a tertiary care hospital in coastal Karnataka. J. Evolution Med. Dent. Sci 2019;8(09):594-598, DOI: 10.14260/jemds/2019/132

\section{BACKGROUND}

Temporomandibular joint is a form of articulation found only in mammals; it is by far the most complex joint in the body. As it provides hinging movement in one plane i.e. forward and backward it is called as ginglymoid joint. However, at the same time it also provides gliding movement which classifies it as an arthrodial joint so known as ginglymoarthrodial joint.[1],[2] The physiologic activities in which the temporomandibular joint plays a part may be voluntary or reflex and ranges from mastication, deglutition, phonation and

'Financial or Other Competing Interest': None.

Submission 08-01-2019, Peer Review 17-02-2019,

Acceptance 22-02-2019, Published 04-03-2019.

Corresponding Author:

Dr. Chethan Hegde,

Head of the Department,

Department of Prosthodontics,

A. B. Shetty Memorial Institute of Dental Sciences,

Derelakatte, Mangalore-575018, Karnataka, India.

E-mail: hegdechethan@yahoo.co.in

DOI: $10.14260 /$ jemds $/ 2019 / 132$

momentary actions such as grasping, blowing and yawning. The joint can undergo various types of changes that might lead to the disorders of the joint, the temporomandibular joint diorder has complex and sometimes controversial aetiologies that might be unknown or undiagnosed in nature,[3],[4] under similar circumstances, one person's temporomandibular joint may appear to deteriorate and degenerate while another does not. However, once degenerative changes start in the Temporomandibular joint, can lead to crippling, and to a variety of anatomical, morphological and functional deformities. Primarily, temporomandibular joint disorders have a non-inflammatory origin, the pathological process is characterized by deterioration and abrasion of articular cartilage and local thickening of the cartilage. There are a heterogeneous group of pathologies that affect the Temporomandibular joint, the masticatory muscles, or both are characterized by a classically described triad of clinical signs, such as muscle and/or Temporomandibular joint pain; Temporomandibular joint sounds; and restriction, deviation, or deflection while wide mouth opening or while doing any kind of functional movements. All these signs go undiagnosed 
and may not be having any symptoms associated with occlusion, masticatory muscles and temporomandibular joint, such as internal or external derangement that might be considered to induce disorder causing dysfunction of the jaw joints.[5],[6] Furthermore, the involvement of parafunctional habits such as bruxism might also be a cause of Temporomandibular joint disorders, while muscular hyperactivity induced by psychological stress and persistently poor oral habits, that might cause muscular fatigue may lead to spasm, resulting in Temporomandibular joint disorders. To rule out all such major causes in asymptomatic individuals that might lead to Temporomandibular joint disorder has to be diagnosed and should be managed to restrict Temporomandibular joint disorder.[7],[8],[9] The present study is to identify the prevalence of signs of temporomandibular joint disorders in asymptomatic young individuals attending tertiary care hospital of a coastal Karnataka.

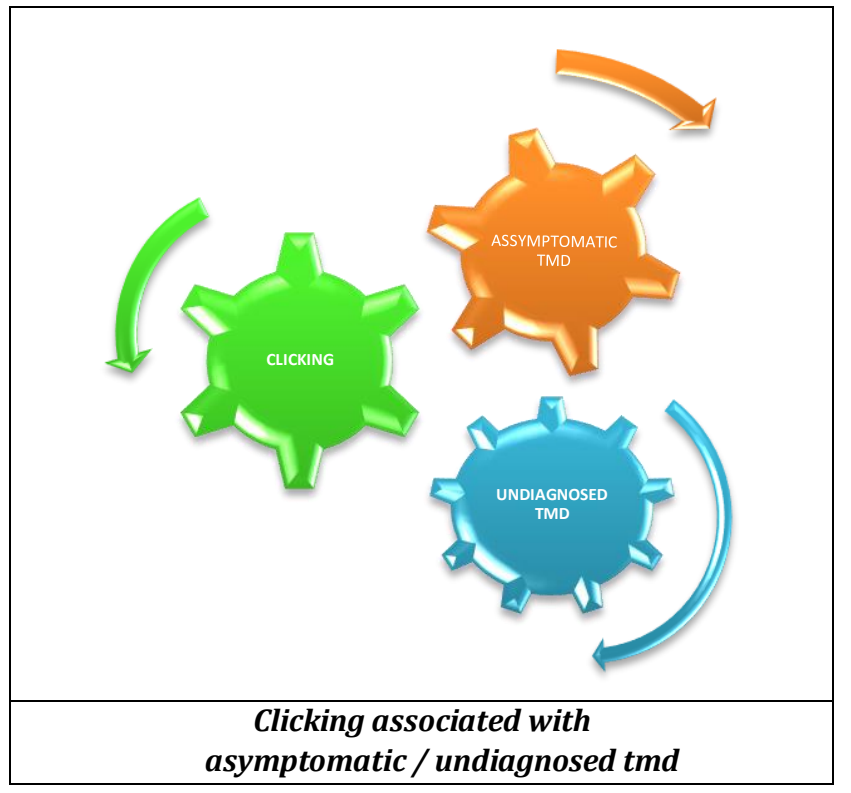

\section{MATERIALS AND METHODS}

\section{Inclusion Criteria}

Healthy young individuals with the age group of 15 to 25 years

\section{Exclusion Criteria}

Subjects with a history of-

A. Head and neck injuries,

B. Temporomandibular joint disorders,

C. Systemic illness,

D. Fractured cases,

E. Neuromuscular disorders

\section{Source of Data Collection}

Based on the results obtained from literature surveys, the prevalence of Temporomandibular joint disorders was found in $25 \%$ of individuals, based on this information the sample size was estimated using the Formulae.

\section{$Z=$ Value of $\alpha$ is $0.05=1.96 \mathrm{P}=25 \mathrm{q}=75 \mathrm{~d}=6$}

\section{Study Design}

Hospital Based Cross Sectional Study.

Sample sizes of 200 healthy adolescents from age group 15 to 25 years will be selected from the Oral Medicine and radiology Department. A. B. Shetty Memorial Institute of Dental Sciences, Deralakatte, Mangalore. The individuals fulfilling the eligibility criteria will be included in the study. Written informed consent will be obtained from each individual. The chosen samples will be clinically examined using auscultation and palpation method based on Research Diagnostic Criteria Index along with a self-report questionnaire which will be given to each individual, the questions will be related to the signs of Temporomandibular joint disorder. The data obtained further will be statistically analyzed using cross sectional study design.[10],[11] Study by convenient sampling technique Since the duration of the study was less. The patients were selected by convenience sampling technique

\section{Clinical Signs Examination}

The signs will be assessed using auscultation and palpation of muscles of mastication (Masseter, temporalis. Medial pterygoid, and lateral pterygoid) based on Research Diagnostic Criteria Index to detect any dysfunction which includes malocclusion, limitation of mandibular movements, clicking, tenderness on the cheek and temporomandibular joint area, deviation and deflection of mouth while yawning and wide opening the mouth.[7],[12],[13],[14] A self-report questionnaire will be used to assess the signs of temporomandibular disordrer, each question will be answered as yes or no.

\section{Statistical Analysis}

The data obtained will be entered in excel sheet and statistically processed, edited, and analysed using descriptive statistics in terms of frequency and percentage were calculated.

\section{RESULTS}

The present study was conducted on a group of 200 subjects to determine the signs of asymptomatic individuals attending tertiary care hospital of a coastal Karnataka; the outcome of the study was as follows.

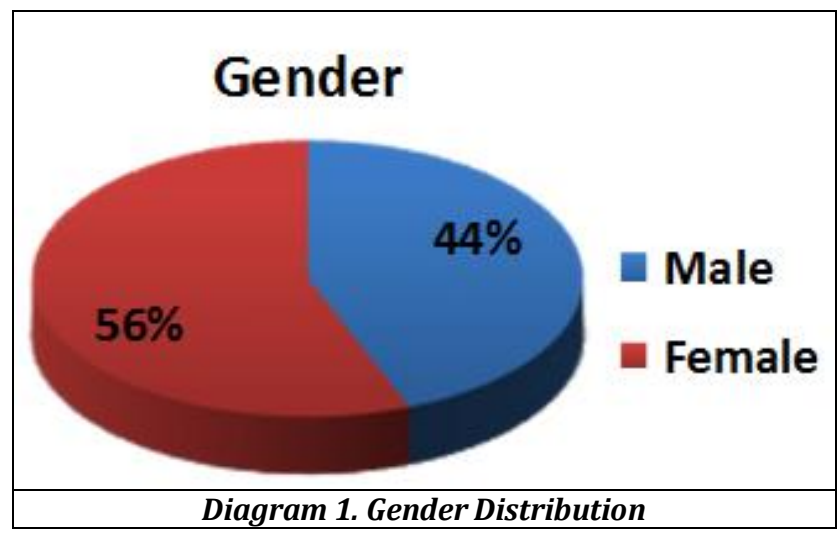

$\frac{\left(Z_{\alpha}\right)^{2} \mathrm{Pq}}{d^{2}}=\frac{3.84 \times 25}{36}=200$ 

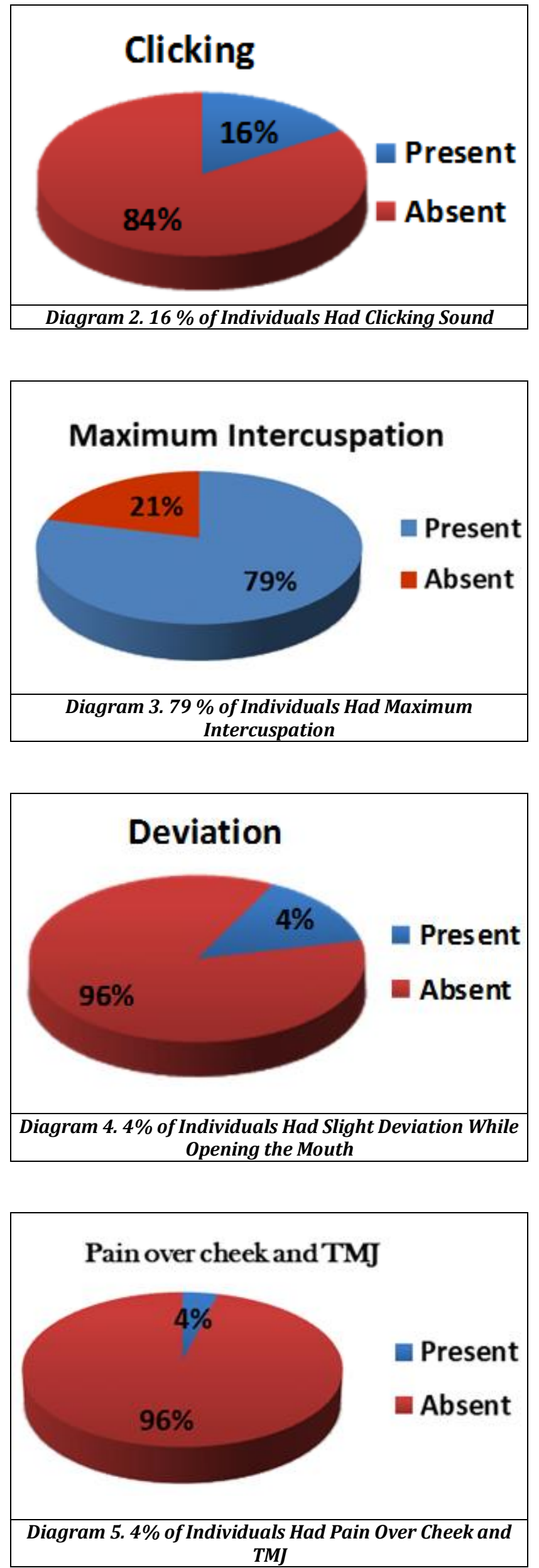

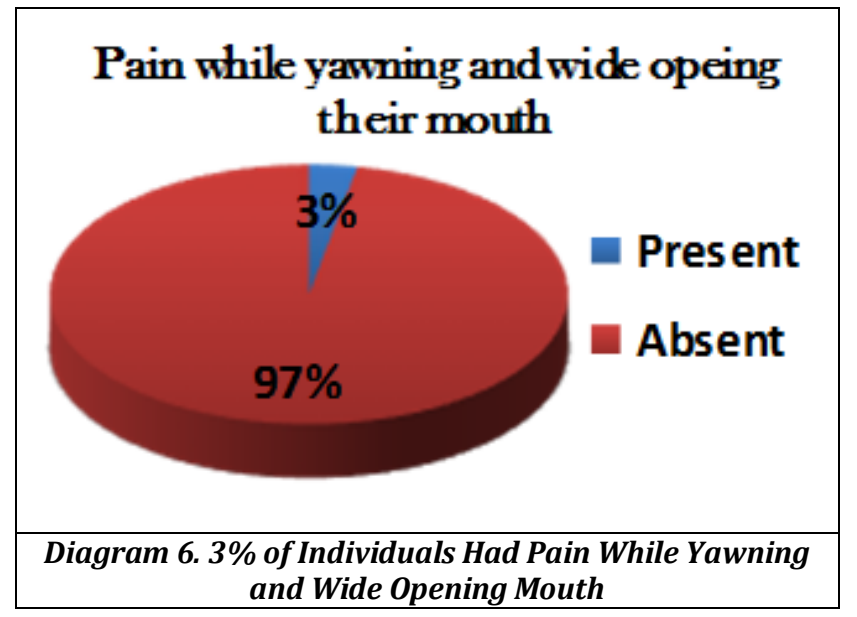

Among the total sample size of 200 subjects, 96 were male (44\%) and 104 were females (56\%). 16\% of individuals had clicking sound, $79 \%$ of individuals had a maximum intercuspation. $4 \%$ of individuals had slight deviation while opening the mouth and had pain over cheek and temporomandibular joint area. $3 \%$ of individuals had pain while yawning and wide opening their mouth. Study by convenient sampling technique since the duration of the study was less. The patients were selected by convenience sampling technique.

\section{DISCUSSION}

The temporomandibular joint is a complex type of articulation that is formed from the articular surfaces of the temporal bone and mandibular condyle. Both the surfaces are layered by dense fibrocartilage. Both the condyles articulate with a large surface area of temporal bone consisting of the articular fossa and articular eminence. The functions of the Temporomandibular joint are unique in that the condyle rotates simultaneously within the fosse and translates anteriorly along the articular eminence. Because of the ability of condyles to translate simultaneously, the mandible can have a much higher maximal incisal opening than would be possible with rotation alone. The joint is thus referred to as "articulation synovialis: a combination of the terms rotation and translation. The maxillo-mandibular relation deformities and temporomandibular joint disorders have been the objective of various studies conducted in literature, and there are various opinions and outcomes of concerning the alteration of temporomandibular joint dysfunction after orthodontic treatment, the trauma of head and neck, carcinoma and surgery. The healthy individuals go unnoticed by various professionals as the temporomandibular joint disorder remains asymptomatic in initial stages. The clinical significance of temporomandibular joint sounds has been a matter of debate for many years; the joint sounds have been extensively investigated with both clinical and instrumental approaches. Temporomandibular joint clicking is commonly recognized as a sign of internal derangement, and its recording is requested to make a diagnosis of disk displacement with reduction according to the most widely adopted classification and diagnostic systems, both in the clinical and in the research settings.[15],[16],[17] The term "TMJ pain" varies greatly in meaning among various health professionals, clinicians, patients, and the general population. Historically, symptombased classification of the disorder has been problematic. As stated by Laskin. The etiological factors that lead to the 
development of Temporomandibular joint disorders can be grouped into three categories and seem to be related to anatomical factors, including occlusion, neuromuscular and psychological factor such as stress, hormonal imbalance, parafunctional activities. Identifying the characteristics of joint sounds in a certain type of occlusal patterns or dental arch configurations may contribute towards the diagnosis of joint pathologies.[18],[19] Nonetheless, the clinical significance of temporomandibular joint click as a pathological sign has been recently questioned in consideration of its weak association with pain and jaw function limitation. ${ }^{[20]}$ Laskin (1969) reported that the etiological factor that cause of temporomandibular joint disorder is muscular hyperactivity induced by abnormal contraction and relaxation of various masticatory muscles, psychological stress and parafunctional oral habits, that may lead to muscular fatigue, causes spasm, and restricted jaw movements resulting in temporomandibular joint dysfunction, further noted that this disorder may cause occlusal disharmony, deformation in articulation leading to alteration in muscular activity. The evaluation and diagnosis of the temporomandibular joint disorder are based on psychological factors and has been considered the very important etiological factor that causes the temporomandibular joint disorder. However, there has been very less number of reports of temporomandibular joint disorders based on psychological factors in population-based studies. There seems to be little literature, that parafunctional habits are involved in temporomandibular joint disorder, but the debate is still in progress to classify whether the parafunctional habits and psychological factors alone or in combination with other factors may lead to Temporomandibular joint disorder. In particular, it is mandatory to conduct a survey to define weather of bruxism and clenching as parafunctional factors may have a major role in patients suffering from the temporomandibular joint disorder. The Significant correlation and association between different signs of temporomandibular joint disorder were formed in the literature review. Instability in the centric relation may elicit motor disturbances in the masticatory input from receptors in the periodontal ligament and Temporomandibular joint. This study evaluated the prevalence of signs of temporomandibular joint disorders in asymptomatic young individuals of a south coastal population of Karnataka. The decision to use a questionnaire and to conduct a physical examination was based on the possibility of objectively measuring the severity of problems in mandibular movements. The symptomatic questionnaire proved to be a simple and suitable tool easily understood by the volunteers. Moreover, the study was carried out in a randomized population and not among people seeking treatment for the temporomandibular joint disorder. Following data was obtained from the present study where clicking sounds were observed in $16 \%$ with $79 \%$ of subjects having maximum intercuspation, deviation and muscle tenderness was observed in $4 \%$ of subjects and pain while yawning and the wide opening of mouth was observed in $3 \%$ of sample size. Based on the data gathered it can be concluded that having maximum intercuspation has no role in avoiding patient suffering from Temporomandibular joint disorder. It was observed that there was a correlation between subjects with maximum intercuspation also had a click, deviation, pain, and muscle tenderness. The Temporomandibular joint disorder may have a treacherous beginning, initially, manifest itself as a click on opening or closing the jaws or mild pain occur while doing various mandibular movements. As discussed earlier the need for treatment may be assessed by the patient themselves or by the clinician while doing a routine clinical check-up. However, it is important to note that most of the population-based studies on temporomandibular joint disorders report a discrepancy between the frequency of symptoms and the frequency of signs of Temporomandibular joint disorders. ${ }^{[24]}$ The temporomandibular joint disorder was significantly associated with occlusal disharmony,

posterior crossbite, malocclusion and extreme cases of overbite and overjet. These morphological disturbances in occlusal disharmony or signs of temporomandibular joint disorder in asymptomatic young individuals may help to treat patients prosthodontically or orthodontically at an early age to eliminate the traits of the anomaly.

In addition, studies have reported varying relationships between subjectively perceived symptoms and signs found upon clinical examination. Since signs of temporomandibular joint disorders obviously make an early appearance, the routine dental examination should include evaluation of these signs and symptoms to identify patients who should be observed more closely.

\section{CONCLUSION}

Based on the results above presented, it was concluded that signs of temporomandibular disorders were present in adolescents. The presence of clinical signs associated with subjective symptoms was confirmed in asymptomatic individuals attending a tertiary care hospital of coastal Karnataka. The temporomandibular joint sounds showed greater prevalence; this finding must be interpreted carefully in order to detect factors that can interfere with proper functioning of stomatognathic system growth and development.

\section{REFERENCES}

[1] Gesch D, Bernhardt O, Mack F, et al. Association of malocclusion and functional occlusion with subjective symptoms of TMD in adults: results of the Study of Health in Pomerania (SHIP). The Angle Orthodontist 2005;75(2):183-90.

[2] Mohlin B, Axelsson S, Paulin G, et al. TMD in relation to malocclusion and orthodontic treatment: a systematic review. The Angle Orthodontist 2007;77(3):542-8.

[3] Kanehira H, Agariguchi A, Kato $\mathrm{H}$, et al. Association between stress and temporomandibular disorder. Nihon Hotetsu Shika Gakkai Zasshi 2008;52(3):375-80.

[4] Thilander B, Rubio G, Pena L, et al. Prevalence of temporomandibular dysfunction and its association with malocclusion in children and adolescents: an epidemiologic study related to specified stages of dental development. The Angle Orthodontis 2002;72(2):146-54.

[5] Tanaka E, Detamore MS, Mercuri LG. Degenerative disorders of the temporomandibular joint: etiology, diagnosis and treatment. Journal of Dental Research 2008;87(4):296-307. 
[6] Manfredini D. Etiopathogenesis of disk displacement of the temporomandibular joint: a review of the mechanisms. Indian Journal of Dental Research 2009;20(2):212-21.

[7] Schiffman E, Ohrbach R, Truelove E, et al. Diagnostic Criteria for Temporomandibular Disorders (DC/TMD) for Clinical and Research Applications: recommendations of the International RDC/TMD Consortium Network* and Orofacial Pain Special Interest Group. J Oral \& Facial Pain Headache 2014;28(1):6-27.

[8] Manfredini D, Basso D, Salmaso L, et al. Temporomandibular joint click sound and magnetic resonance-depicted disk position: Which relationship? Journal of dentistry 2008;36(4):256-60.

[9] Barkin S, Weinberg S. Internal derangements of the temporomandibular joint: the role of arthroscopic surgery and arthrocentesis. Journal Canadian Dental Association 2000;66(4):199-203.

[10] Hayashi T, Ito J, Koyama J, et al. The accuracy of sonography for evaluation of internal derangement of the temporomandibular joint in asymptomatic elementary school children: comparison with MR and CT. American Journal of Neuroradiology 2001;22(4):728-34.

[11] Dujoncquoy JP, Ferri J, Raoul G, et al. Temporomandibular joint dysfunction and orthognathic surgery: a retrospective study. Head \& Face Medicine 2010;6(1):27.

[12] Ozkan A, Altug HA, Sencimen M, et al. Evaluation of articular eminence morphology and inclination in TMJ internal derangement patients with MRI. Int J Morphol 2012;30(2):740-4.
[13] Garcia AR, Zuim PR, Goiato MC, et al. Effect of occlusion on joint sounds in asymptomatic individuals. Acta Odontologica latinoamericana: AOL 2008;21(2):13540.

[14] Salé H, Isberg A. Delayed temporomandibular joint pain and dysfunction induced by whiplash trauma: a controlled prospective study. The Journal of the American Dental Association 2007;138(8):1084-91.

[15] Sato S, Sakamoto M, Kawamura H, et al. Disc position and morphology in patients with nonreducing disc displacement treated by injection of sodium hyaluronate. International Journal of Oral \& Maxillofacial Surgery 1999;28(4):253-7.

[16] American Society of Temporomandibular Joint Surgeons. Guidelines for diagnosis and management of disorders involving the temporomandibular joint and related musculoskeletal structures. Cranio 2003;21(1):68-76.

[17] Padala S, Padmanabhan S, Chitharanjan AB. Comparative evaluation of condylar position in symptomatic (TMJ dysfunction) and asymptomatic individuals. Indian Journal of Dental Research 2012;23(1):122.

[18] Goiato MC, Garcia AR, Dos Santos DM, et al. TMJ vibrations in asymptomatic patients using old and new complete dentures. Journal of Prosthodontics: Implant, Esthetic and Reconstructive Dentistry 2010;19(6):43842.

[19] Larheim TA, Westesson PL, Sano T. Temporomandibular joint disk displacement: comparison in asymptomatic volunteers and patients. Radiology 2001;218(2):428-32.

[20] Herb K, Cho S, Stiles MA. Temporomandibular joint pain and dysfunction. Current Pain and Headache Reports 2006;10(6):408-14. 